\title{
Computation Pre-Processing Techniques for Image Restoration
}

\author{
Aziz Makandar \\ Professor \\ Department of Computer Science, \\ Karnataka State Women's University, Vijayapura
}

\author{
Anita Patrot \\ Research Scholar \\ Department of Computer Science, \\ Karnataka State Women's University, Vijayapura
}

\begin{abstract}
Image restoration is to enhance the image quality which is blurred and noised from various defects which damage the quality of an image. The most degradation is done in motion blur and noise defects as shown in the results. This introduces and implements the computing methods used in the image processing world to restore images as well as improve the quality by threshold. In order to know the detailed information carried in the digital image for better visualization. The aim is to provide information of image degradation and restoration process by various filters such as wiener filter, blind convolution and wavelet techniques are used in experiments in this paper will be presented as followed by MATLAB simulation results. Weiner filter gives maximum PSNR value and minimum MSE value in $\mathrm{dB}$ comparable to other techniques for image restoration.
\end{abstract}

\section{General Terms}

Image Processing, Restoration, Pre-processing.

\section{Keywords}

Blurring, Noise, Weiner, Blind Convolution, Wavelet, PSNR, MSE, RMSE

\section{INTRODUCTION}

The idea of image restoration is to minimize the noise [5,2] and blurring image [4,2] from a degraded image by various atmospheric defects. It introduces an effective method for image restoration is de-convolution based on a coefficient of image formulated in the wavelet domain wavelet based denoising of an image can be done since Donoho in [9]. Regularizations achieved by promoting a reconstruction with wavelet multi resolution expressed in the wavelet coefficients, taking advantage of the well known decomposition of wavelet representations. The results of the suppressing image degradation using knowledge about its nature. There are two groups such as deterministic methods and stochastic techniques. The original image is obtained from the degraded one by transformation inverse to the degradation [2]. Stochastic techniques the best restoration is shown according to some stochastic criterion in digital image processing textbooks in chapter 4 they explained the techniques of preprocessing, e.g., a least squares method. In some cases the degradation transformation must be estimated first. It is advantageous to know the duration function explicitly. The restoration results of Matlab show the better knowledge of the image, are the result of the restoration as discussed in [3]. The Image preprocessing can also called as image restoration, involves the corrections of atmosphere deflects, degradation and noise introduced during the imaging process. This process produces a corrected image that is as close as possible characteristics of the original image.

\section{IMAGE RESTORATION AND PREPROCESSING}

Image restoration uses a priori knowledge of the degradation. It models the degradation and applies inverse process. It formulates and evaluates the objective criteria of goodness. The distortion can be modeled as noise or a degradation function. The formulation of the problem is to enhance the image quality by applying various restoration methods as discussed above and removes the noise as well as blurring from the degradation function, then removes the noise and blur through the Wiener filter, blind convolution function and wavelet based restoration. Here $f(n, m)$ is an input image, $\wedge^{\wedge} f$ $(\mathrm{n}, \mathrm{m})$ restoration function.

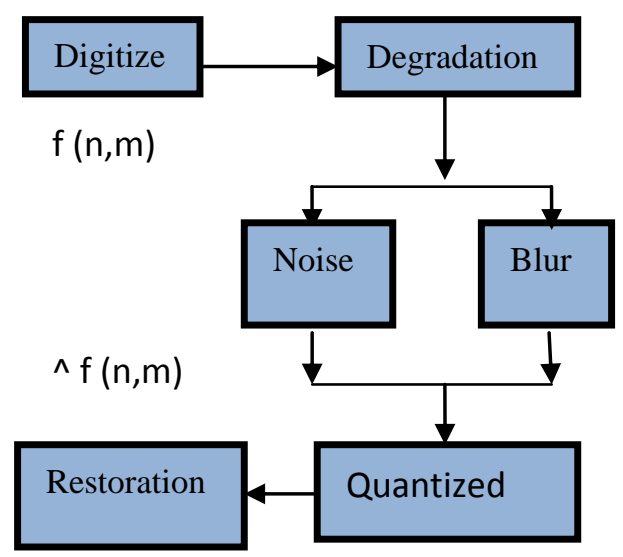

Figure 1: Formulation of problem

To restore an image from linear degradation various filters are used such as inverse, pseudo inverse, wiener filter and blind de-convolution are used in various techniques. Image restoration is the operation of taking a corrupted or noisy image and estimate the clean of the original image. Degradation may come in many forms such as motions blur and noise. Subspace analysis on blurred image in [3] and blind convolution in [4] for a linear invariant system, the observed or distorted image I $(\mathrm{x}, \mathrm{y})$ can be modeled as a convolution of the object function o $(\mathrm{x}, \mathrm{y})$ which is the actual object in the scene with the image degradation function $h$ $(\mathrm{x}, \mathrm{y})$ which also commonly known as the point spread function.

$$
\mathbf{I}(\mathbf{x}, \mathbf{y})=\mathbf{o}(\mathbf{x}, \mathbf{y}) * * \mathbf{h}(\mathbf{x}, \mathbf{y})+\mathbf{n}(\mathbf{x}, \mathbf{y})
$$

Where $n(x, y)$ is an additive noise function that describes the random variation of the pixel intensity of an image. The convolution theorem is used. 


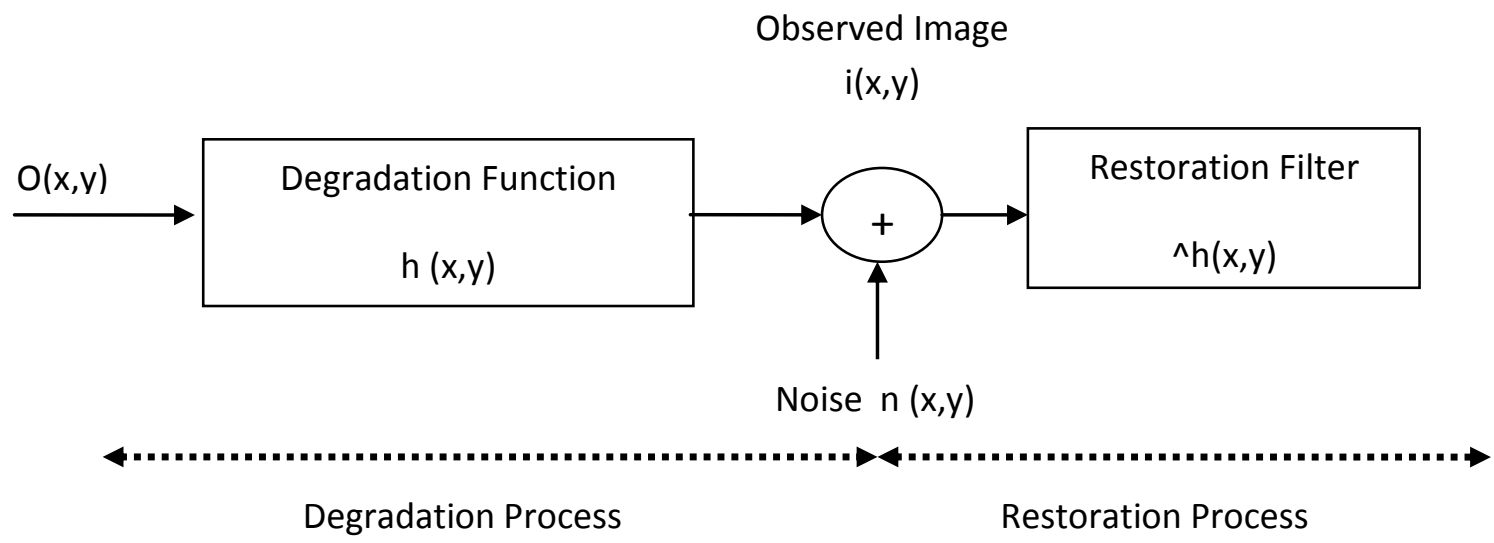

Figure 2: Degradation Process Model

Fourier transforms in the frequency domain. Thus, the image degradation model can be written as

$$
\mathbf{I}(\mathbf{u}, \mathbf{v})=\mathbf{O}(\mathbf{u}, \mathbf{v}) \mathbf{H}(\mathbf{u}, \mathbf{v})+\mathbf{N}(\mathbf{u}, \mathbf{v})
$$

In a simplest image degradation model is discussed in two types of filters one is a low pass filter and the second one is high pass filters built in degradation modeled as a low pass filter which resulted in a blur effect. Figure. 2 show the block diagram of image degradation and restoration process. The image restoration process involves restoration filter ${ }^{\wedge} \mathrm{h}(\mathrm{x}, \mathrm{y})$ in reversing the distortion effects. As shown in the block diagram of image degradation is added noise to the original image and restore. Simultaneously degradation process and restoration process are done with a degraded image from various filters as we discussed in this paper, Weiner filter, blind convolution and wavelet based filters are used on noised image and restore the image.

The image restoration process can be achieved by enforcing the image degradation process, i.e.,

$$
\widehat{\mathbf{0}}(\mathbf{u}, \mathbf{v})=\frac{\mathbf{I}(\mathbf{u}, \mathbf{v})-\mathbf{N}(\mathbf{u}, \mathbf{v})}{\mathbf{H}(\mathbf{u}, \mathbf{v})}=\frac{\mathbf{I}(\mathbf{u}, \mathbf{v})}{\widehat{\mathbf{H}}(\mathbf{u}, \mathbf{v})}
$$

Where $\frac{1}{\widehat{H}(u, v)}$ The inverse is filtered, and $\widehat{O}(u, v)$ is the recovered image. Although the concept is relatively simple, the actual implementation is difficult to achieve, as one requires prior knowledge or identifications of the unknown degradation function $\mathrm{h}(\mathrm{x}, \mathrm{y})$ And the unknown noise source $n(x, y)$.

\section{RESTORATION TECHNIQUES}

\subsection{Noise Models}

Digital images are prone to a variety of noises. The noise result is nothing but some information is missing from the image acquisition process that result in pixel values that do not reflect the true intensities of the original image. The ways that noise can be introduced into an image, depending on how the image is created. The term impulse noise is also used for this type of noise discussed in [2]. Other terms are spike noise, random noise or independent noise. Black and white dots appear in the image explained in [5] as a result of this noise and hence salt and pepper noise. This noise arises in the image because of sharp and sudden changes of the image signal.

\subsection{Blur Models}

The image can make or become unclear or less distinct is known as blur. Motion blur occurs when there is relative motion between the object and the camera during exposure. This can be in the form of translation, rotation, and sudden change of scale or atmospheric turbulence occurs due to random variations in the reflective index of the medium between the object and the imaging system and it occurs in the imaging of astronomical objects.

\subsection{Weiner Filter}

The effect of noise distribution has not been considered in the inverse filter operation. Let the additive noise power spectrum be $S_{n n}(u, v)$ and image power spectrum be $S_{x x}(u, v)$. Generally, $S_{n n}(u, v)$ has a dominant effect over $S_{x x}(u, v)$ in the high frequency region, as $S_{x x}(u, v)$ Tends to concentrate in the low frequency spectrum. In the Fourier domain, the Wiener filter is expressed as

$$
\mathbf{W}(\mathbf{u}, \mathbf{v})=\frac{\mathbf{H}^{*}(\mathbf{u}, \mathbf{v})}{|\mathbf{H}(\mathbf{u}, \mathbf{v})|^{2}+\mathbf{S}_{\mathrm{nx}}(\mathbf{u}, \mathbf{v})}
$$

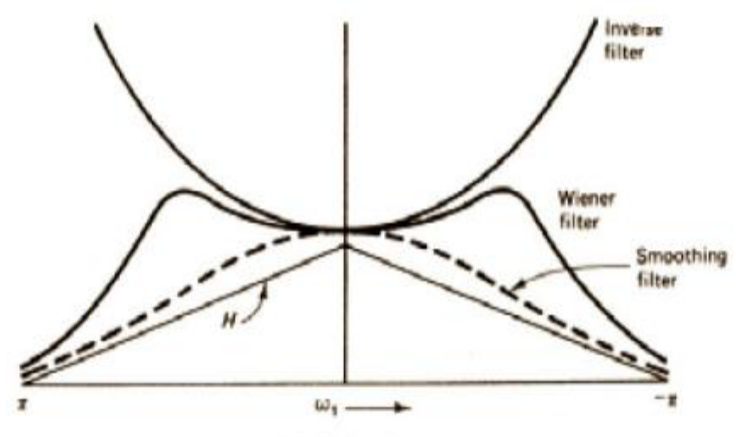

(b) Deblurring

Figure 3: Computation of Weiner and Inverse Filter.

Where $S_{n x}(u, v)=S_{n n}(u, v) / S_{x x}(u, v)$ is the noise-to-signal ratio. One can see that in the high frequency region the resulting $S_{n x}(u, v)$ Will be relatively large, i.e., $S_{n x}(u, v) \gg$ $|\mathrm{H}(\mathrm{u}, \mathrm{v})|$. Consequently, the high frequency response of the restoration filter is suppressed. Figure 3, shows the power spectrum characteristics of the inverse filter and Wiener filter.

It is worth noting that if no noise is presented, i.e. $S_{n n}(u, v) \rightarrow$ 0 , the Wiener filter is the inverse filter:

$$
\left.\mathbf{W}(\mathbf{u}, \mathbf{v})\right|_{\mathbf{S}_{\mathrm{nn}}(\mathbf{u}, \mathbf{v}) \rightarrow \mathbf{0}}= \begin{cases}\frac{1}{\mathbf{H}(\mathbf{u}, \mathbf{v})}, & \mathbf{H}(\mathbf{u}, \mathbf{v}) \neq \mathbf{0} \\ \mathbf{0}, & \mathbf{H}(\mathbf{u}, \mathbf{v})=\mathbf{0}\end{cases}
$$




\subsection{Median Filter}

The This example shows how to remove salt and pepper noise from an image using an averaging filter and a median filter (medfilt2) to allow comparison of the results. The filtering is similar to an averaging filter in that each output pixel is set with an average of the pixel values in the neighborhood of the corresponding input pixel. However, with filtering, the value of an output pixel of an image is determined by the median of the neighbor pixels rather than the mean. The median is much less sensitive than the mean to tremendous values. Median filtering is one of the most accurate filtering technique used in image processing therefore better able to remove these outlets without reducing the sharpness of the image.

\subsection{Blind Convolution}

Both inverse and Wiener filter requires accurate estimation of the degradation function and knowledge about the noise model for image restoration. The blind de-convolution is a method where accurate estimation of the degradation function is not required because of an initial guess of the degradation function is sufficient for understanding of an image. The iterative process of blind de-convolution, which is usually carried out in the frequency domain the blind de-convolution procedure not only attempt to restore the blurred image, but also estimate the degradation function.

Computing H (f1, f2) computes Svv (f1, f2) from observing image and by assuming Suu $(\mathrm{f} 1, \mathrm{f} 2)$ known $\log |\mathrm{H}| 2=\log (\mathrm{Svv}-$ Snn)- $\log$ Suu. This does not give the phase of the $\mathrm{H}$, in many cases $\mathrm{H}$ is linear phase, e.g. due to motion blur or zero phase.

\subsection{Wavelet based Restoration}

The wavelet based restoration technique is introduced in this technique [6] is specially used to reduce the noise from the image with loss of little information from the image. The idea of wavelet denoising based on the assumption that the amplitude rather than the location of the spectra of the signal to be as different as possible for that of noise.

The DWT is used to discretely distributed based on frequency as well as time domain. In wavelet transforms, the original image is divided into frequency resolution and time contents the decomposition of the image intensity is two level discrete wavelet transform as shown in the figure. 5

The wavelet based image restoration is specially used to diagnose the image which is degraded by the atmospheric or unknown defects. After decomposition of the image by using two discrete wavelet transform is represented in figure. 5 in this illustrates the function of decomposition in levels and we are getting LL, LH, HL, and HH information from the image as shown in figure. 5 The $2 \mathrm{dwt}$ function is used to get high frequency as well as time intensity contents from the image by using a low pass filter and high pass filter for getting accurate.

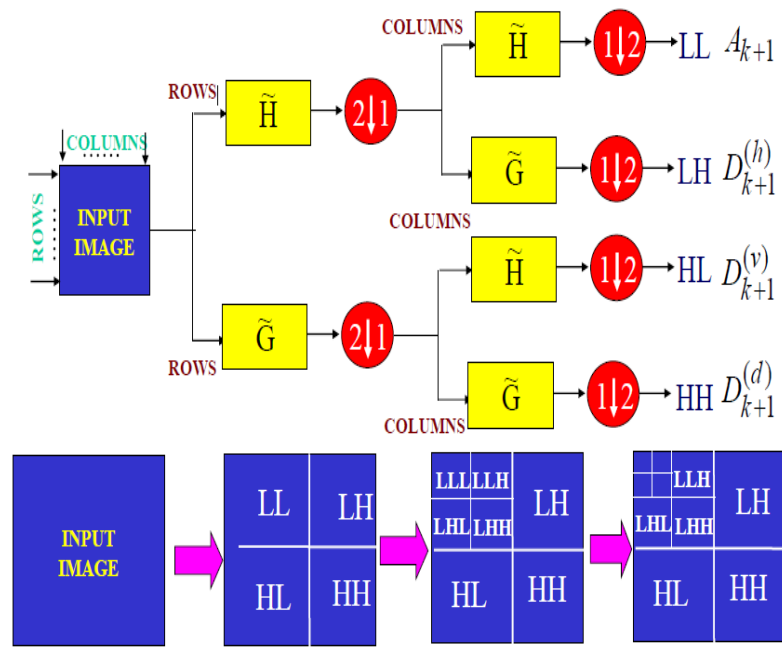

Figure 4: Decomposition of Image using wavelet.

The high pass filter and low pass filter are used to get the row wise and column wise information from the image as we know the low pass filter is used to smoothen the image and high pass filter is used to sharpening the edges of an image.
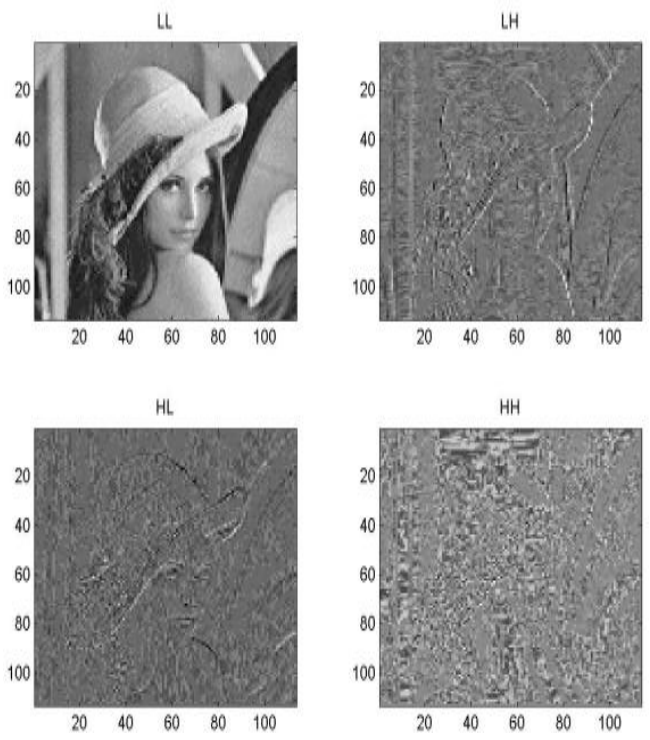

Figure 5: Implementation of 2DWT

\section{RESULTS}

The The reluctant images are as shown in the following figure which consists of original images, noised image and blurred image by applying restoration techniques like Weiner filter, blind convolution and wavelet based restoration.

The figure 1 shows the blind convolution which includes original image, then by adding motion blur to the original image, de-blurring with undersized PSF, de--blurring with oversized PSF and reconstructed weighted array as shown in the simulation results of restoration techniques. 


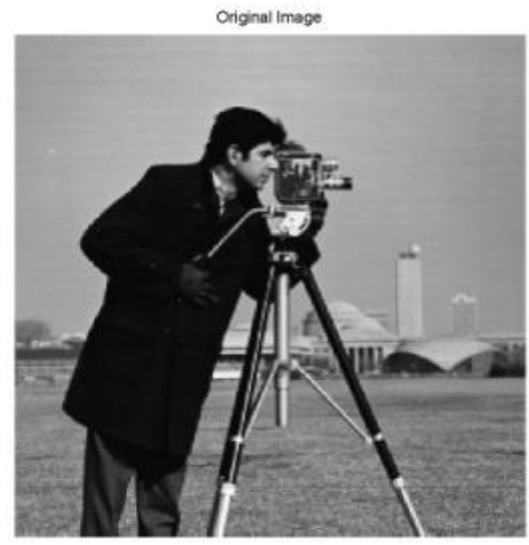

(a) Original Image

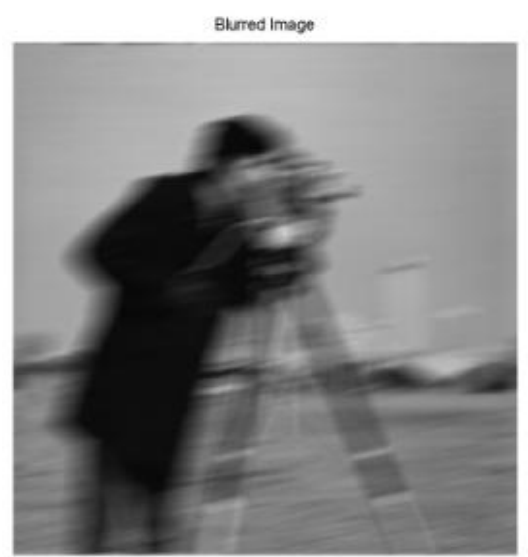

(b) Blurred Image

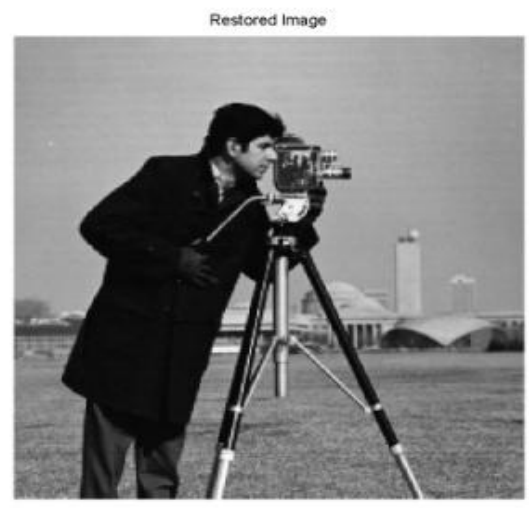

(c) Restored Image

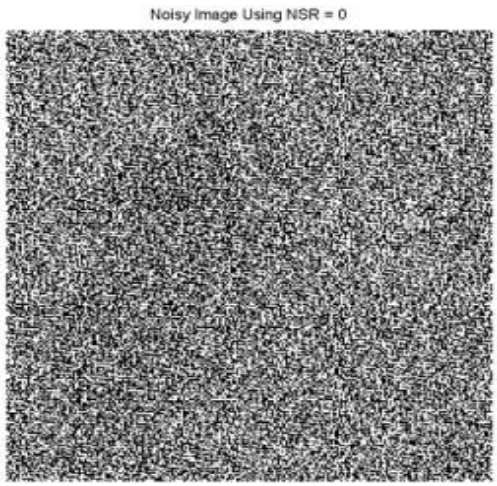

(d) Noisy Image using NSR=0

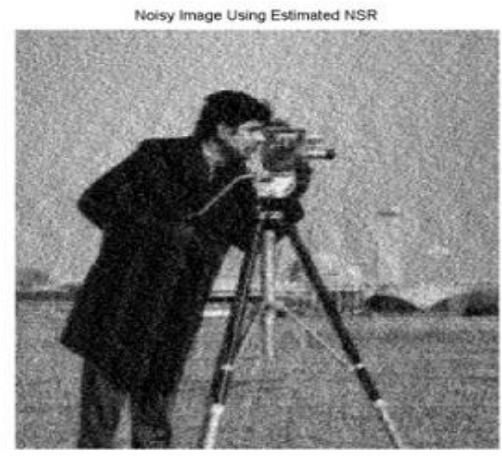

(e) Simulate Blur and Noise

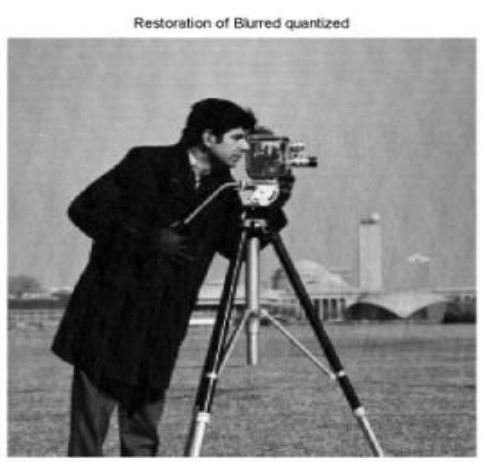

(f) Restored Image

Figure 6: Weiner Filter Simulation Results for Blurred Image to Restore Image

The Figure 6, shows the steps followed in the Weiner filter with noise and blur of a cameraman image is tested for various degradation functions and pre-processing techniques for better quality image enhancement as well as clarity of an image to visualize. (a) Original Image (b)Blurr Image (c)Restored Image (d)Noisy Image using NSR=0 (e) Simulate Blur and Noise (f) Restored Image all as shown in the above figure.

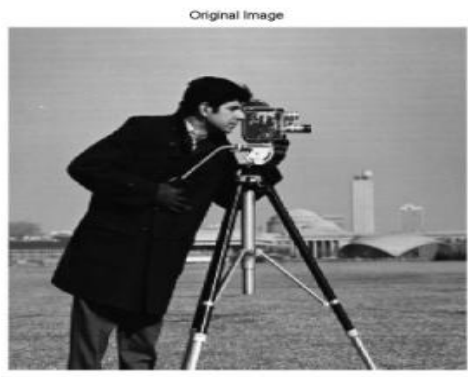

(a) Original Image

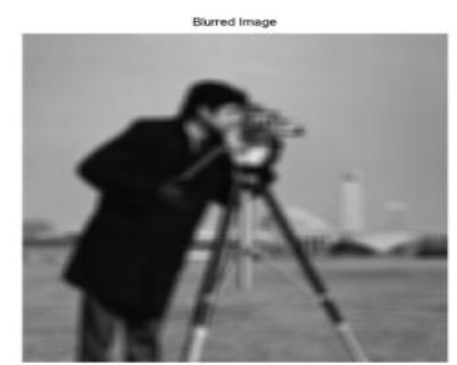

(b) Blurred Image 


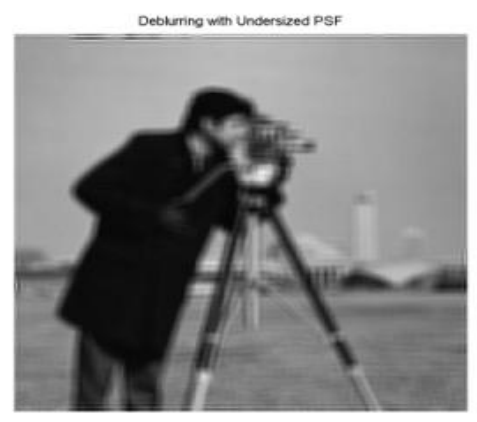

(c) Deblurring With PSF

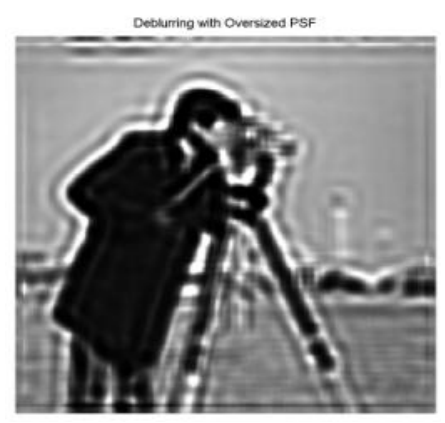

(d) Deblurr with over PSF

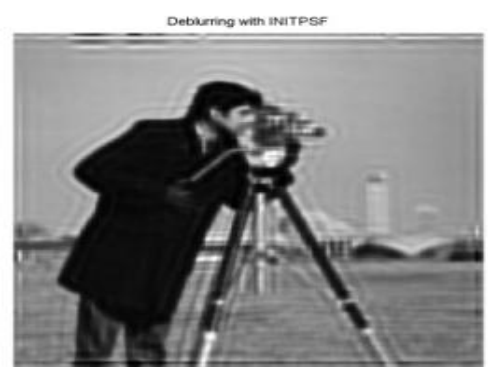

(e) Deblurr with TSPF

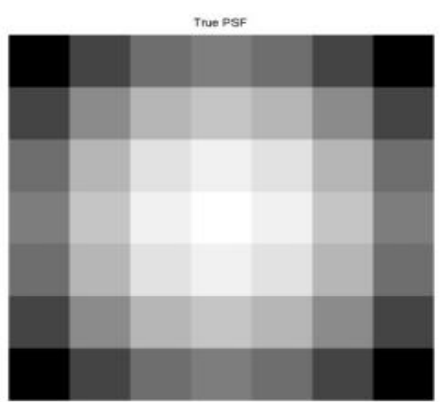

(f) True PSF

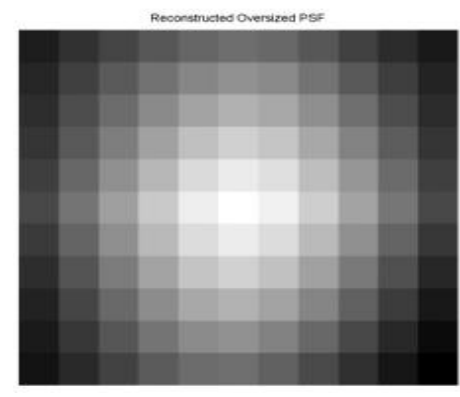

(g) Reconstructed Over PSF

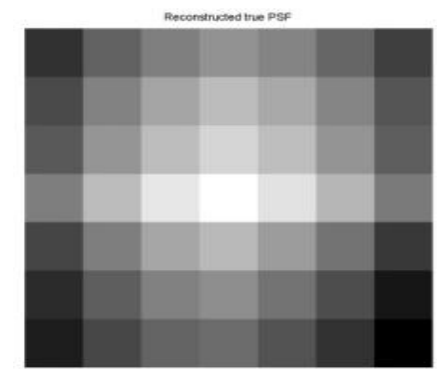

(h) Reconstructed True SPF

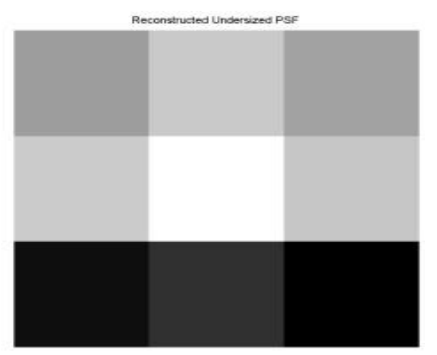

(i) Reconstructed Undersized PSF

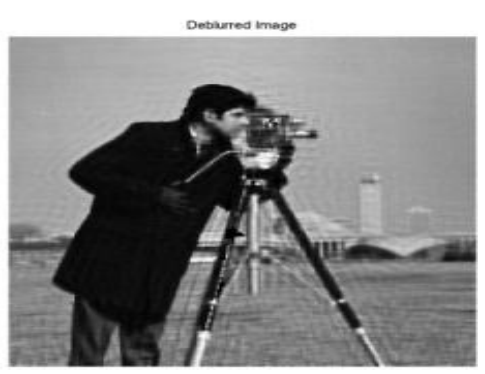

(j) Restored Image

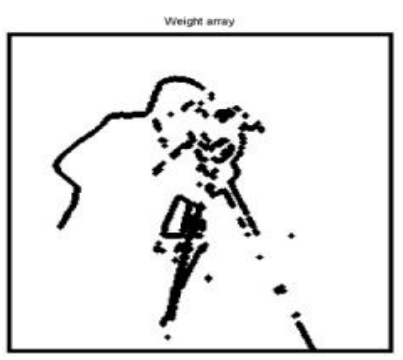

(k) Weight Array

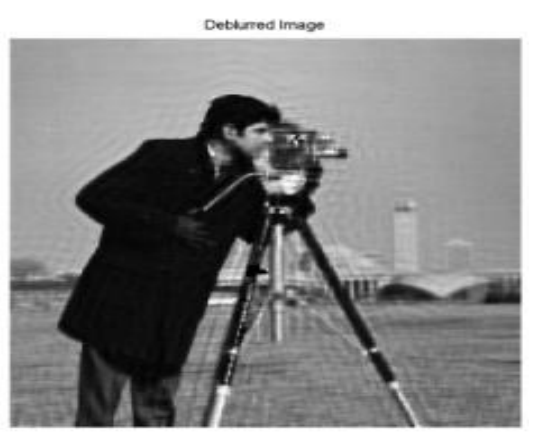

(l) Restored image

Figure 7: Blind-Convolution Function 
The Figure 7, shows the steps followed by the blind convolution function which gives the above results such as noise and blur of a cameraman image. (a) Original Image (b) Blurr Image (c) Deblurring with PSF (d) Deblurr with over PSF (e) Deblurr with TSPF (f) True PSF (g) Reconstructed over PSF (h) Reconstructed True SPF (i) Reconstructed undersized PSF (j) Restored Image (k) Weight array.

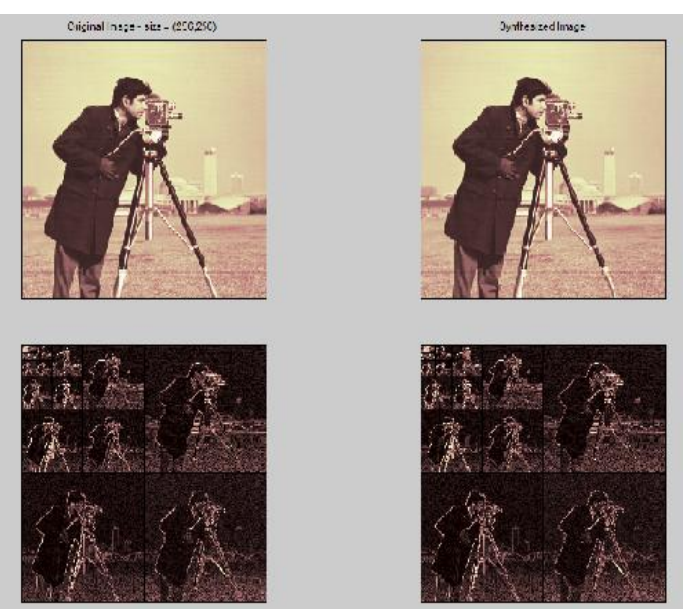

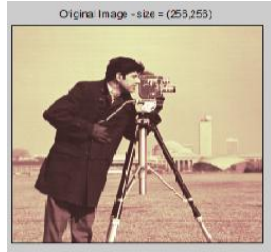
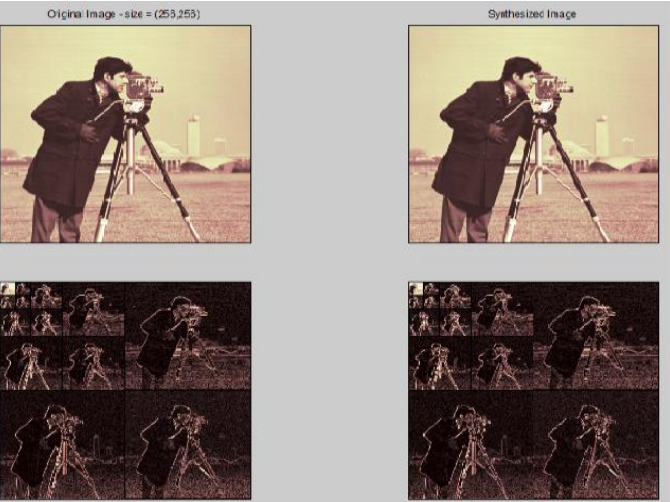

Figure 8: Bi orthogonal filter and Haar wavelet.

Table 1: Comparison of Quality Measures.

\begin{tabular}{|c|c|c|c|}
\hline Techniques & MSE & RMSE & PSNR \\
\hline Weiner & 0.0 & 0.02 & $83.79 \mathrm{~dB}$ \\
\hline $\begin{array}{c}\text { Blind } \\
\text { Convolution }\end{array}$ & 24.42 & 4.94 & $34.29 \mathrm{~dB}$ \\
\hline Wavelet & 22.42 & 4.74 & $34.69 \mathrm{~dB}$ \\
\hline
\end{tabular}

Weiner

Blind Convolution

Wavelet

Blind Convolution

Weiner

PSNR

Figure 9: Computation of Quality Measures.

\section{CONCLUSION}

This paper described methods for reducing noise as well as for reducing blur, and for the combination of these two of the images. But primarily an image restoration is done mostly using Weiner filter, Richardson-Lucy Blind Deconvolution algorithm, Inverse and Pseudo-inverse filter. Image restoration remains for nonlinear algorithms most of the literature focuses either on the de-noising problem or blurring problem considers both blur and noise simultaneously. This paper focus on the combination of both noise and blur of the image still appears that just consider the blur without treating the noise, but these are the exception to aware of the instabilities of de-convolution and realize that one must simultaneously consider the effects of noise when trying to remove blur to get more enhanced image from degradation process.

\section{FUTURE WORK}

Image restoration is an active research area and various researchers work to improve the efficiency of the different algorithms by developing more efficient algorithms. There are several application domains of image restoration like scientific exploration, legal investigations, film making and archives, image and video decoding and consumer photography. The main area of application is an image reconstruction in radio astronomy, radar imaging and tomography $[1,2]$. This paper discusses the importance of image restoration techniques and reviews different image restoration techniques available.

\section{REFERENCES}

[1] Menu Poulose,'Literature Survey on Image Blurring Techniques," International Journal of Computer Application Technology and Research, vol. 2, Issue. 3, pp. 286-288, 2013.

[2] Biswa Ranjan. Mohapatra. Ansuman Mishra. Sarat Kumar Rout,"A Comprehensive Review on Image Restoration Techniques," International Journal of Research in Advent Technology, Vol. 2, no. 3, March 2014-ISSN: 2321-9637.

[3] Nishiyama, M. Hadid. A. Takeshima H. Shotton, J. Kozakaya T. And Yamaguchi, O.2011 Facial de-blur inference using subspace analysis for recognition of 
blurred faces, IEEE Trans. Pattern Anal. Mach. Intell, vol. 33 , no. 4

[4] Kondor. D. and Hatzinakos,D. Blind Image deconvolution revisited.

[5] Hu. H. and Haan, G. 2006 Low cost, robust blurs estimator Proc. IEEE Int'l Conf. Image Processing, pp. 617-620.

[6] Mario A. T. Figueiredo, et.al,"An EM algorithm for Wavelet- Based Image Restoration," IEEE Trans. Image Processing, Vol. 12, No. 8, August 2003.

[7] Aziz Makandar, Anita Patrot, and Bhagirathi Halalli," Color Image Analysis and Contrast Stretching using Histogram Equalization,". International Journal of Advanced Information Science and Technology (IJAIST). ISSN 2319:2682 Vol.27, No.27, July 2014.

[8] D. L. Donoho, "De-noising by soft-thresholding," IEEE Trans. Information Theory, Vol.41, no. 3, pp. 613-627, May 1995.
[9] S. Grace Chang. Bin Yu and M. Vattereli, "Adaptive Wavelet Thresholding for Image Denoising and Compression," IEEE Trans. Image Processing, Vol. 9, pp. 1532-1546, Sept. 2000.

[10] Mrs. C. Mythili and Dr. V. Kavitha. Efficient Technique for Color Image Noise Reduction $\mathrm{T}$ h e $\mathrm{R}$ e s e a $\mathrm{r} \mathrm{c} \mathrm{h} \mathrm{B} \mathrm{u}$ 11 e t I n o f J o r d a n ACM, V o l. I I (I I I)

[11] L. Yang and J. Ren, "Remote sensing image restoration using estimated point spread function", 2010 .International Conference on Information, Networking and Automation (ICINA), IEEE, 2010.

[12] D. Maheswari et. al. Noise Removal in Compound Image using median filter. (IJCSE) International Journal of Computer Science and Engineering Vol. 02, No. 04, 2010, 1359-1362

[13] C. Solomon and T. Breckon, 'Fundamentals of Digital Image Processing,' John Wiley \& Sons, Ltd, 2011. 